\title{
Die Bedeutung des Parameters Zeit bei der Behandlung des fortgeschrittenen Hörsturzes
}

\subsection{Einleitung}

\subsubsection{Hinführung zum Thema}

Auch wenn beim Hörsturz über eine hohe Rate an Spontanremissionen berichtet wird, findet man in der Literatur immer wieder Hinweise darauf, dass die Heilungsaussichten bei dieser Erkrankung umso günstiger ausfallen, je rascher nach seinem Auftreten mit einer Behandlung begonnen worden ist [30, 33, 57, 78, 97, 101]. Über den Zeitverlauf der Erholung existieren bislang aber nur vergleichsweise wenige Publikationen [15, 22, 25, 33, 61].

\subsubsection{Zeitphasen der Hörentwicklung beim Hörsturz}

Die Entwicklung des weiteren Hörvermögens nach dem Hörsturzereignis setzt sich aus vier verschiedenen Zeitphasen (Abb. 3.1) zusammen. In diesem Kapitel sollen die einzelnen Intervalle, so weit es geht, in ihrer Bedeutung für die Gesamterholung näher unter die Lupe genommen werden. Dies v. a. auch unter dem Aspekt, herauszufinden, welche Zeiträume optimalerweise für den Einsatz der einzelnen Therapieoptionen genutzt werden sollten, welche „Wartezeiten“ nach dem Hörsturzereignis bis zur Durchführung der explorativen Tympanoskopie dabei in Kauf genommen werden können und ab welchem Zeitpunkt nach Abschluss der letzten Behandlungsmaßnahme mit großer Wahrscheinlichkeit davon ausgegangen werden kann, dass als Folge des Hörsturzes und seiner Behandlung weitergehende Veränderungen der Hörsituation nicht mehr zu erwarten sind, also von einer dauerhaften, langfristig verbleibenden Hörsituation ausgegangen werden kann. 


\subsubsection{Ablauf der Untersuchungen und Therapien}

Eine erste audiologische Befunderhebung ist naturgemäß erst unmittelbar in Zusammenhang mit der stationären Aufnahme der Patienten möglich (Abb. 3.1). Fälschlicherweise (Begründung s. Abb. 3.1) bezeichnen wir das Hörvermögen zum Zeitpunkt der Aufnahme in unsere Klinik im Weiteren als „Ausgangs- oder Anfangshörverlust". Im Mittel war in unserem Patientengut jedoch bereits ein Zeitintervall von 2,76 Tagen (Variationsbreite 0-7 Tage) zwischen dem Hörsturzereignis und der Krankenhausaufnahme verstrichen. Es folgten dann nach einer mehr oder weniger langen Periode eine systemische Kortikoidtherapie (Prednisolon ${ }^{\circledR}$; anfangs i.v.-Applikation, später oral) nach dem Stennert-Schema unter zusätzlicher Gabe von $500 \mathrm{mg} / \mathrm{d}$ in 0,9 \%iger NaCl-Lösung weitere audiologische Kontrolluntersuchungen bis zu einem individuell bestimmten und nicht an eine Regel gebundenen Termin der Tympanoskopie (Mittelwert: 5,27 Tage zwischen Klinikaufnahme und Operation; Variationsbreite: 1-16 Tage) [62]. Es wurden mehrere tonschwellenaudiometrische Untersuchungen, teilweise noch unter stationären Kautelen, bis zum 20. Tag nach dem chirurgischen Eingriff vorgenommen. Eine letzte Hörmessung fand im Rahmen einer Kontrolluntersuchung bei erneuter Einbestellung der Patienten in die Klinik statt (Mittelwert: 2,3 Jahre).

Präoperativ wurden die Audiogrammergebnisse vom Zeitpunkt der stationären Aufnahme ausgehend in folgende drei Gruppen zur Auswertung aufgegliedert:

- 0-2. Tag nach der stationären Krankenhausaufnahme,

- 3.-5. Tag nach der stationären Krankenhausaufnahme,

- ab 6. Tag nach der stationären Krankenhausaufnahme (keine Grenzziehung nach oben).

Nach der Tympanoskopie erfolgte auch eine Zusammenfassung der Hörmessdaten, diesmal in vier Zeitintervallen:

- 1.-3. postoperativer Tag,

- 4.-6. postoperativer Tag,

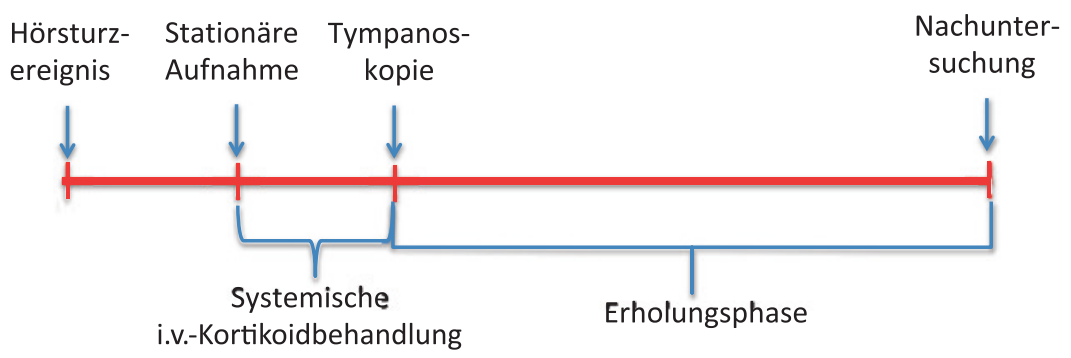

Abb. 3.1 Zeitphasen während der Behandlung eines Hörsturzes mit i.v.-Kortikoidbehandlung und Tympanoskopie inkl. Obliteration der runden/ovalen Fensternische 
- 7.-10. postoperativer Tag und

- 10.-20. postoperativer Tag.

Die für die Auswertung gebildeten, langfristigen Zeitperioden wurden unterteilt in:

- 21.-182. postoperativer Tag (erstes Halbjahr nach der Tympanoskopie),

- 183.-365. postoperativer Tag (zweites Halbjahr nach der Tympanoskopie),

- 366.-730. postoperativer Tag (2. Jahr nach der Tympanoskopie) und

- >731. postoperativer Tag nach der Tympanoskopie (ab 3. Jahr nach OP).

Die Nachuntersuchung lag im Mittel 2,2 Jahre (Variationsbreite: 22 Tage bis 12,9 Jahre) nach dem Hörsturzereignis. Die nach den oben angegebenen Zeitintervallen untergliederten Auswertungsgruppen ab dem 21. postoperativen Tag bildeten zusammengenommen die Basis für die Berechnung des langfristigen Hörkurvenverlaufes, der im Rahmen der Nachuntersuchung erfasst worden war.

\subsubsection{First- und Second-Line-Behandlungsmodus}

Es wurde u. a. auch der Frage nachgegangen, ob früh Tympanoskopierte (FirstLine-Modus) sich in ihrem Hörgewinn von den Patienten unterschieden, bei denen der Operation eine längere systemische Kortikoidbehandlung vorausgegangen war (Second-Line-Modus). Als Frühoperierte (TYMP-FL) wurden dabei jene Patienten eingestuft, bei denen der stationäre Krankenhausaufenthalt vor Durchführung des chirurgischen Eingriffs nicht mehr als 3 Tage und die Zeit zwischen Hörsturzereignis und Operation maximal 5 Tage betragen hatte $(n=10)$. Bei Erkrankten, bei denen die Tympanoskopie im Second-Line-Mode (TYMP-SL) erfolgt war, hatte die stationäre Aufnahme, ebenso wie natürlich auch das Hörsturzereignis, schon mehr als 1 Woche (im Mittel 11,6 Tage) seit Eintritt der akuten Hörminderung zurückgelegen $(\mathrm{n}=10)$.

Eine Aufgliederung in eine Gruppe der First- und Second-Line-Operierten war insbesondere für eine Vergleichbarkeit unserer Ergebnisse mit anderen veröffentlichten Studienresultaten von Bedeutung.

\subsection{Ergebnisse}

Befunde in Zusammenhang mit dem Hörsturzgeschehen

\subsubsection{Präoperativer Beobachtungszeitraum}

\subsubsection{Präoperative Hörentwicklung}

Es zeigte sich, dass innerhalb der ersten 6 Tage nach der stationären Aufnahme trotz der in diesem Zeitraum erfolgten systemischen Kortikoidtherapie noch keine 
Veränderungen im mittleren Hörvermögen eingetreten waren, sich insbesondere auch keine Hörgewinne eingestellt hatten (Abb. 3.2).

\subsubsection{Langfristige postoperative Hörentwicklung}

\subsubsection{Hörgewinn in Abhängigkeit vom Zeitpunkt der Tympanoskopie}

Der Hörgewinn stand in unserem Patientengut in keinem statistisch relevanten Verhältnis zum Zeitintervall, das zwischen dem Hörsturzereignis und dem Termin der Tympanoskopie vergangen war $(r=0,173 ; n=42)$ (Abb. 3.3, linker Abbildungsteil). Auch 11-16 Tage nach dem Eintritt der Hörminderung konnten noch deutliche Hörgewinne erreicht werden (37,2 dB; $n=16$ ) (Abb. 3.3, rechter Abbildungsteil).

\subsubsection{Empfehlungen zum Zeitpunkt der Tympanoskopie}

Der Zeitpunkt, wann die Tympanoskopie angesetzt werden sollte, wird in der Literatur kontrovers diskutiert. Die in unserer Studie fehlende Korrelation zwischen der Dauer des der Operation vorausgehenden Zeitintervalls und dem Hörgewinn steht im Gegensatz zu den Befunden von Grossenbacher et al., Reineke et al. wie auch Strohm et al., die fanden, dass ein frühzeitiges chirurgisches Vorgehen gewählt werden sollte [25, 78, 87]. Strohm et al. konnten in einer Untersuchung, die einen Zeitraum von mehr als 300 Tagen nach der Operation beinhaltete, eine exponentiell abfallende Rate von Hörverbesserungen nach Eintritt des Hörsturzes beobachten [87].

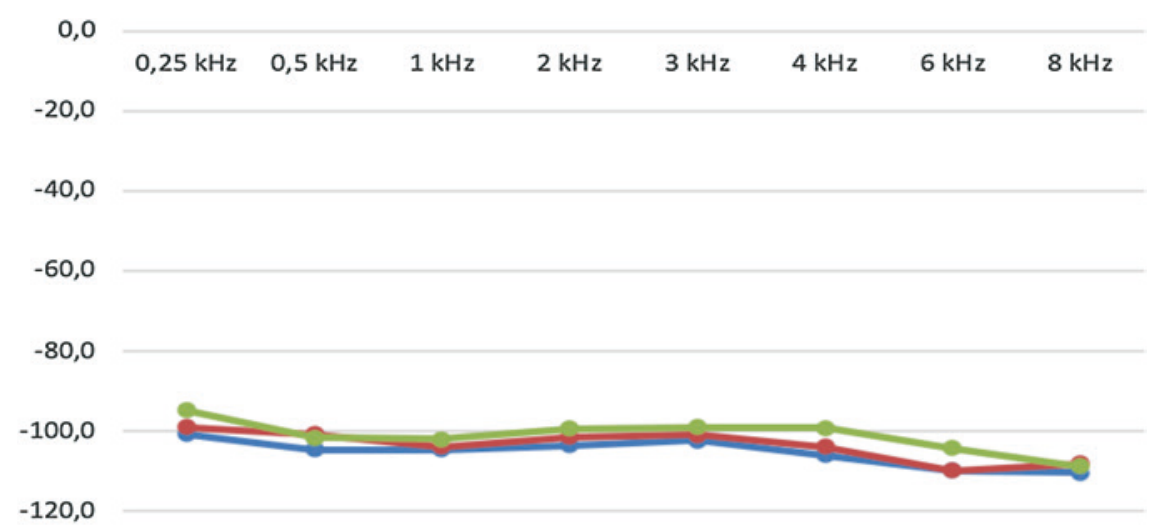

AW - 1.-3. Tag präoperativ $\simeq 4 .-6$. Tag präoperativ

Abb.3.2 Präoperative Hörentwicklung von der stationären Krankenhausaufnahme (mittlere Anfangshörverluste) bis zum Tympanoskopietermin. AWAusgangshörverlust; Abszisse: gemessene Frequenzen in kHz; Ordinate: Tonhörverluste in $\mathrm{dB}$ 


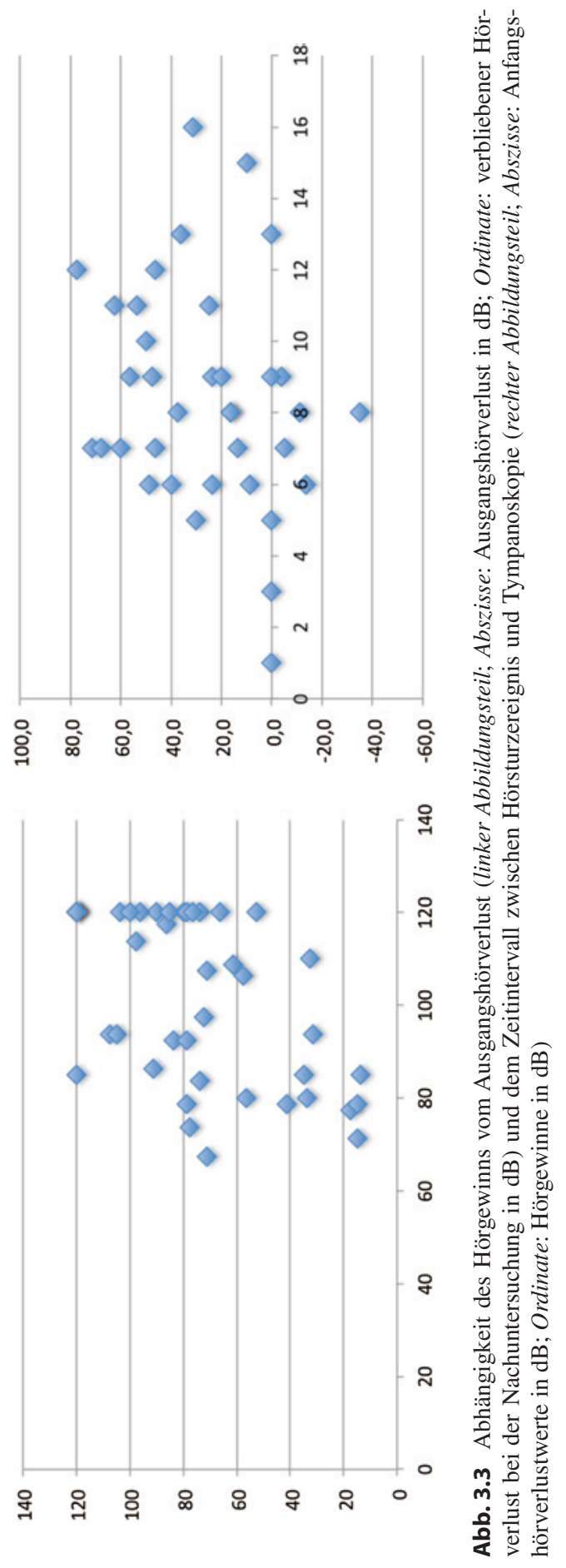


Kampfner et al., Hoch et. al., wie auch Reineke et al. und Thomas et al. konnten in ihren Patientenpopulationen jeweils signifikante Unterschiede im Ausmaß des Hörgewinns nach der Operation in Abhängigkeit von der Tatsache feststellen, ob die Erkrankten frühzeitig nach dem Hörsturzereignis oder erst verzögert zur stationären Krankenhausaufnahme und damit auch der Mittelohroperation gekommen waren [30, 33, 78, 97]. Bei Thomas et. al. war das Merkmal „Latenzzeit zwischen Hörsturzereignis und Operation“" sogar der einzige Faktor, der sich neben vielen anderen von ihm überprüften Parametern im Rahmen einer multivariaten Analyse seiner Daten als signifikant prognoserelevant hinsichtlich des Hörgewinns herausgestellt hatte [97]. Dabei korrelierte eine längere Latenzzeit jeweils mit schlechteren Hörgewinnen.

Andere Autoren hingegen favorisieren den verzögert indizierten Eingriff [22, 43, 61]. So äußerten Gedlicka et al., dass man den operativen Eingriff möglichst nicht $\mathrm{zu}$ früh nach dem Hörsturzereignis durchführen sollte, um dem Patienten die Chance einer Spontanremission nicht zu nehmen [22]. Darüber hinaus gaben sie zu bedenken, dass die Operation ja doch mit einer Traumatisierung verbunden ist [22, 43, 61]. Aufgrund unserer Ergebnisse, dass auch nach längerer systemischer Behandlungszeit mit Kortikosteroiden noch recht respektable Hörerholungen eingetreten sind, können wir uns der Auffassung dieser zuletzt genannten Gruppe überzeugter anschließen. Unsere Beobachtungen scheinen dem Kliniker also Zeit einzuräumen, bei seinen Patienten zunächst einmal den Erfolg einer konservativen Behandlung abwarten zu können, bevor er zu dem Mittel des chirurgischen Eingriffs greift. Aufgrund unserer Erfahrung setzt er sich mit einem abwartenden Vorgehen hinsichtlich der Indikationsstellung zur Tympanoskopie wohl keinem Vorwurf eines Versäumens wertvoller, ungenutzt verstrichen gelassener Zeit aus.

\subsubsection{Hörgewinne im Gesamtpatientengut}

Insgesamt unterschieden sich in unserem Patientengut die mittleren Tonhörverluste, die zu Beginn der stationären Behandlung (100,8 dB; SAW: 17,2 dB; n=37) ermittelt wurden, hochsignifikant $(\mathrm{p}<0,001)$ von denen, die nach durchschnittlich 2,2 Jahren Nachbeobachtungszeit erhoben wurden (73,9 dB; SAW: 30,8 dB; $\mathrm{n}=37)$.

\subsubsection{Hörerholung First- und Second-Line-Operierter}

Die Hörerholung in den Gruppen der First- und Second-Line-Operierten stellte sich bei unseren Patienten unterschiedlich dar (Abb. 3.4). Die Frühoperierten zeigten im Mittel, bezogen auf den PTA $_{4}$-Wert, einen Hörgewinn von 32,9 dB (SAW: 34,9 dB; $n=10)$, während dieser bei den Spätoperierten mit 40,7 dB (SAW 22,3 dB; $n=10$ ) sogar deutlich stärker ausgeprägt war. Dieser Unterschied lag statistisch aber deutlich oberhalb der 5-\%-Irrtumswahrscheinlichkeit $(p=0,56)$. Dies traf gleichermaßen auch auf die verbliebenen Endhörverluste $(\mathrm{p}=0,61)$ zwischen der Gruppe der Frühoperierten (FL-Gruppe) und der Spätoperierten (SL-Gruppe) zu, die keine signifikant unterschiedlichen mittleren Hörmesswerte zum Zeitpunkt der Nachuntersuchung boten. 


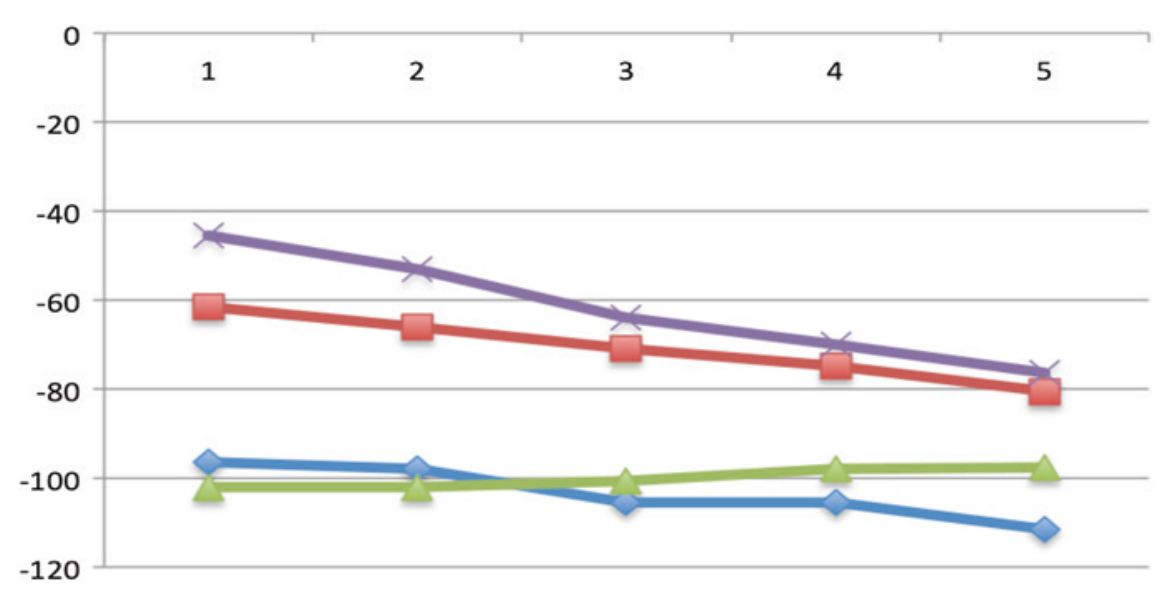

Abb. 3.4 Anfangs- und Endhörverluste in der Gruppe der Patienten, die frühzeitig $(<4$ Tage, FL-Gruppe) und verzögert (>10 Tage, SL-Gruppe) nach dem Hörsturzereignis tympanoskopiert worden waren. Abszisse: geprüfte Tonhörverluste in kHz; Ordinate: Anfangs- und Endhörverluste in dB. Der oberste violette Kurvenzug (bessere Restitution des Hörvermögens) entspricht der Hörerholung bei Patienten, bei denen die Tympanoskopie mit mehr als 10 Tagen Verzug nach dem Hörsturzereignis stattgefunden hatte, der darunter liegende (geringere Restitution des Hörvermögen) dem von Patienten, die innerhalb der ersten 3 Tage nach Behandlungsbeginn mit systemischen Kortikosteroiden behandelt worden waren. Unterer Bildpunkt: Anfangshörverlust; oberer Bildpunkt: Resthörverlust bei der Nachuntersuchung

Vergleicht man in der Subpopulation der im First-Line-Modus Operierten die Untergruppe, die langfristig Hörgewinne von $\geq 30 \mathrm{~dB}$ erzielen konnte, mit derjenigen, in der nur Hörerholungen von $<30 \mathrm{~dB}$ erreicht wurden, so ergaben sich bezüglich der Geschlechtszusammensetzung, der Verteilung der Seitenlokalisation des erkrankten Ohres, dem Durchschnittsalter, dem Vorliegen einer Vorerkrankungen auf dem betroffenen Ohr, der Durchführung einer ambulante Vortherapie, dem Vorliegen einer Auslösesituation oder von Schwindel bzw. Tinnitus zeitgleich zum Eintritt der Hörminderungen, und der Tatsache, ob intraoperativ ein pathologischer Mittelohrbefund nachgewiesen wurde, keine sich auf dem Signifikanzniveau abgesicherten Unterschiede $(n=10)$. Damit ist aufgrund unserer Beobachtungen bezüglich epidemiologischer und anamnestischer Daten keine Vorhersage möglich, welches Patientenkollektiv bei dem frühen Einsatz der Tympanoskopie bessere Hörgewinne durch die Therapie erwarten lässt.

Unsere Ergebnisse zeigen aber auch, dass im First-Line-Modus Therapierte im Einzelfall zu beachtlichen Hörgewinnen kommen können (bis zu 71,2 dB).

\subsubsection{Literaturangaben zum Hörgewinn}

Auch im Literaturvergleich, in den nur 20 Patienten aus unserem Krankengut einfließen konnten, bestätigte sich, dass die kombiniert (systemische Kortikoidapplikation plus Tympanoskopie) Behandelten, also früh tympanoskopierten Patienten, mit einem geringeren Hörverlustlevel $(94,1 \mathrm{~dB} ; \mathrm{n}=79)$ gegenüber der Gruppe der verzögert Operierten $(104,2 \mathrm{~dB} ; \mathrm{n}=247)$ ihre Therapie begannen. Die Frühoperierten zeigten dann geringere Hörgewinne in der Folgezeit, als es 
bei den verzögert chirurgisch Behandelten (Frühoperierte: Hörgewinn 23,4 dB; Späteroperierte: 38,1 dB) der Fall war. Die langfristig gemessenen mittleren Höreinschränkungen betrugen bei den früh tympanoskopierten Patienten gemittelt noch 70,7 dB, bei den verzögert Operierten 66,1 dB. Wegen des Zusammentragens der Daten aus verschiedenen Publikationen konnten diese Werteunterschiede keiner statistischen Auswertung mit Überprüfung auf Signifikanzunterschiede unterzogen werden. Diese Ergebnisse unterstützen nach unserer Meinung also die bereits oben beschriebenen Empfehlungen, nach denen die Tympanoskopie mit einem größeren Erfolg verbunden ist, wenn sie nicht zu früh nach dem Hörsturzereignis vorgenommen wird.

\subsubsection{Frequenzbezogene Hörgewinne}

Vom Zeitpunkt der stationären Aufnahme bis zum Nachuntersuchungstermin boten die Patienten unseres Krankengutes mit unauffälligem intratympanalen Befund frequenzabhängig unterschiedliche Hörverbesserungen (Abb. 3.5). Die tiefen Frequenzen erholten sich, von nahezu gleichen Ausgangswerten ausgehend, im Mittel deutlich besser als die hohen. Die Summe der mittleren Hörverluste in den Frequenzen 0,25 und $0,5 \mathrm{kHz}$ betrug initial $100,2 \mathrm{~dB}$, die der Endhörverluste 65,0 dB (Hörgewinn 35,2 dB). Demgegenüber lag die Summe der mittleren Hörverluste bei den Tonhöhen 4 und $8 \mathrm{kHz}$ anfangs bei 106,7 dB. Hier wurden dann Endhörverlust von im Mittel 88,8 dB erreicht (Hörgewinn $16,9 \mathrm{~dB})$. Die Anfangshörverluste $(\mathrm{p}=0,03)$, mehr aber noch die Endhörverluste

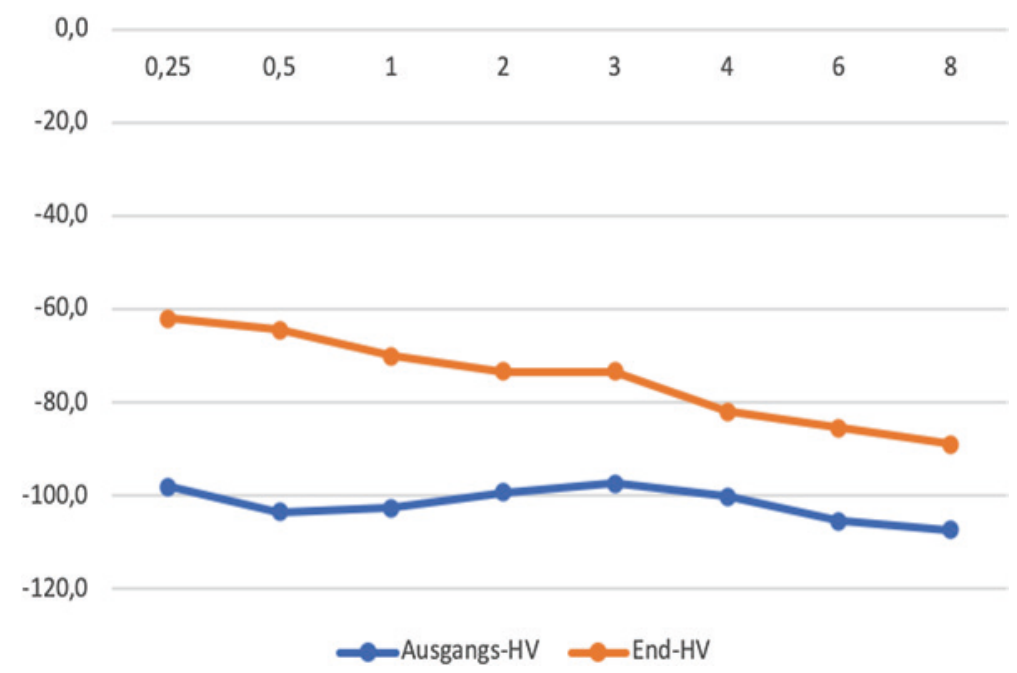

Abb. 3.5 Mittlere Ausgangs- und Endhörverluste, durchschnittlich 2,3 Jahre nach Hörsturz. Abszisse: Gemessene Tonhöhenfrequenzen in kHz; Ordinate: Hörverluste (HV) in $\mathrm{dB}$. Blaue Kurve: Anfangshörverlust; orange Kurve: Resthörverlust bei der Nachuntersuchung 
$(\mathrm{p}<0,001)$, unterschieden sich zwischen dem Tief- und Hochtonbereich dabei jeweils statistisch signifikant.

\subsubsection{Unterschiedliches Hörerholungsverhalten in Einzelfällen}

In den ersten Tagen nach dem operativen Eingriff kann es einerseits zunächst zu weiteren Hörverschlechterungen kommen, bis sich dann Hörverbesserungen einstellen (Abb. 3.6, rechter Abbildungsteil). Andererseits beobachteten wir auch einzelne Patienten, bei denen sich schon sehr früh nach der Tympanoskopie bessere Hörkurven, als sie bei der letzten Audiogrammkontrolle unmittelbar präoperativ gemessen wurden, eingestellt hatten (Abb. 3.6, linker Abbildungsteil).

Diese Beobachtung sollte nach unserer Meinung dazu beitragen, zu verhindern, dass sich der Operateur auch bei anfänglichen weiteren Verschlechterungen des Gehörs unmittelbar postoperativ dazu hinreißen lässt, an einem langfristig guten Ergebnis der Operation zu zweifeln, oder dass er evtl. sogar vorschnell einen Revisionseingriff vornimmt.

\subsubsection{Zeitlicher Ablauf der postoperativen Hörerholung bis zum 20. postoperativen Tag}

Postoperativ setzten bei den von uns Behandelten die ersten deutlichen Zeichen einer Hörerholung im Mittel zwischen dem 10. und 20. Tag nach Mittelohreingriff ein, wobei sich die Messwerte statistisch unter Verwendung der mittleren Hörverluste $\left(\mathrm{pTA}_{4}\right)$ nicht als signifikant unterschiedlich gegenüber den Werten bestätigten, die noch zwischen dem 7.-9. Tag nach Tympanoskopie registriert worden waren $(p=0,17)$ (Abb. 3.7, linker Abbildungsteil). Diskrete Hörverbesserungen hingegen ließen sich im Tieftonbereich in den durchschnittlichen Hörverlustwerten schon unmittelbar nach dem chirurgischen Eingriff erkennen.

Basierend auf zwei Studien zeigte sich, dass der zum Zeitpunkt der Tamponadeentfernung aus dem äußeren Gehörgang ermittelte Hörverlust noch nicht dem langfristig erreichbaren entspricht ([33] und eigenes Patientengut). Nach der ersten postoperativen Woche war mit einem Hörgewinn von $21,4 \mathrm{~dB}$, ausgehend von einem Hörverlust von 101,6 dB, eine Hörminderung von $79,2 \mathrm{~dB}$ erreicht, was erst $65,5,0 \%$ (Hörgewinn: $22,4 \mathrm{~dB}$ ) der gesamten, auf längere Zeit erreichten Hörverbesserung entsprach (Endhörverlust: 66,9 dB; Hörgewinn: 33,7 dB). Im eigenen Patientengut war nach im Mittel 10,25 Tagen nach OP nur ein Hörgewinn von 19,0 dB aufgetreten, das entspricht 47,5\% des Gesamthörerfolges $(40,5 \mathrm{~dB} ; \mathrm{n}=18)$ nach 2,3 Jahren.

\subsubsection{Hörentwicklung nach dem 22. postoperativen Tag}

In verschiedenen Subpopulationen von Patienten unseres Gesamtkrankengutes, bei denen Nachuntersuchungen zu jeweils unterschiedlichen Zeiten mit mehr als 


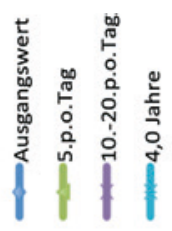

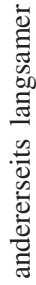

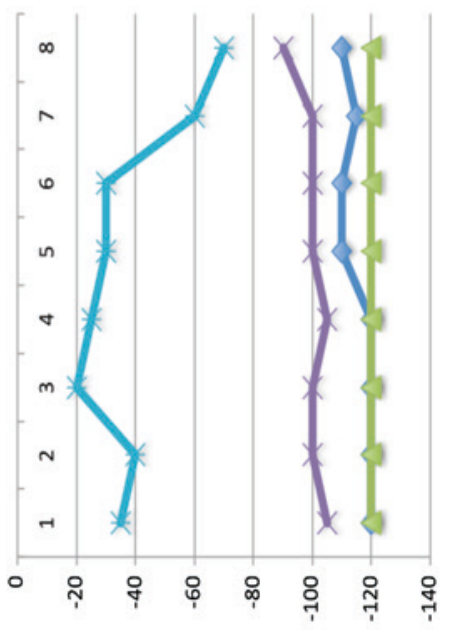

$\bar{\Xi}$

:

o.

吾

$\stackrel{\nabla}{\frac{\pi}{8}}$

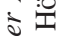

章

$\dot{0}: \frac{1}{2}$

$\frac{\overline{0}}{\overline{0}}$

总 空

를

ह

늘

范

.

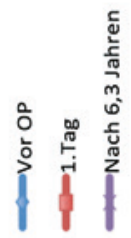

엥

三

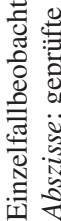

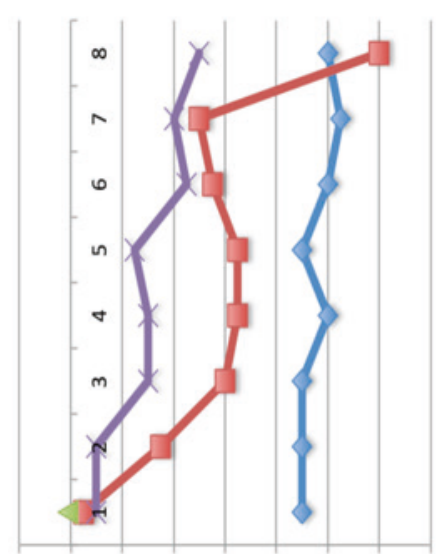

$\circ$ ๆ

ए

N

$\ddot{\otimes}$

号

‡

突

至

ป气

壱 :

然

등

कृ

:어 $: \frac{1}{8}$

0

mi 

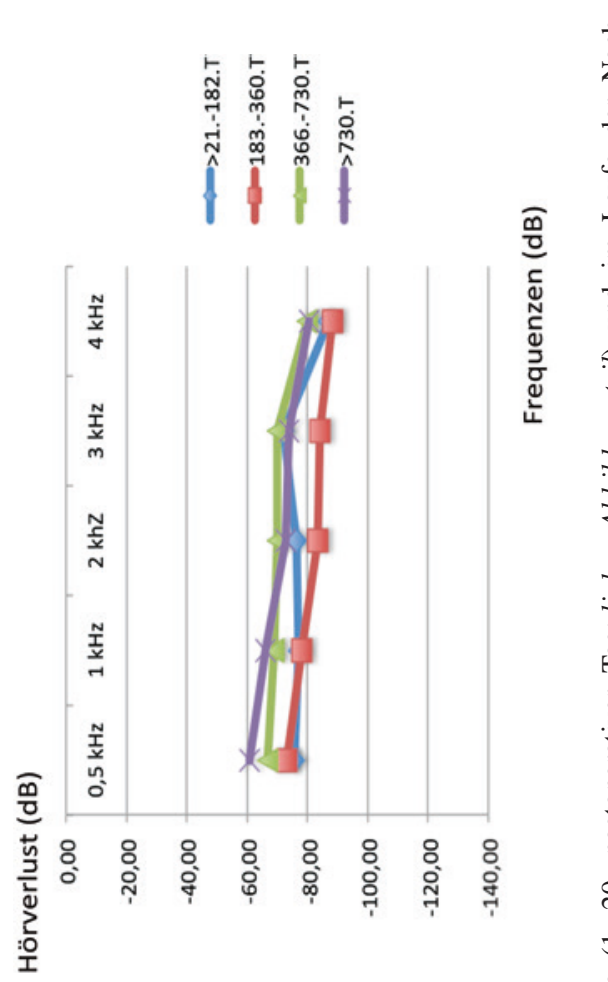

$\frac{1}{0}$

鸟

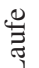
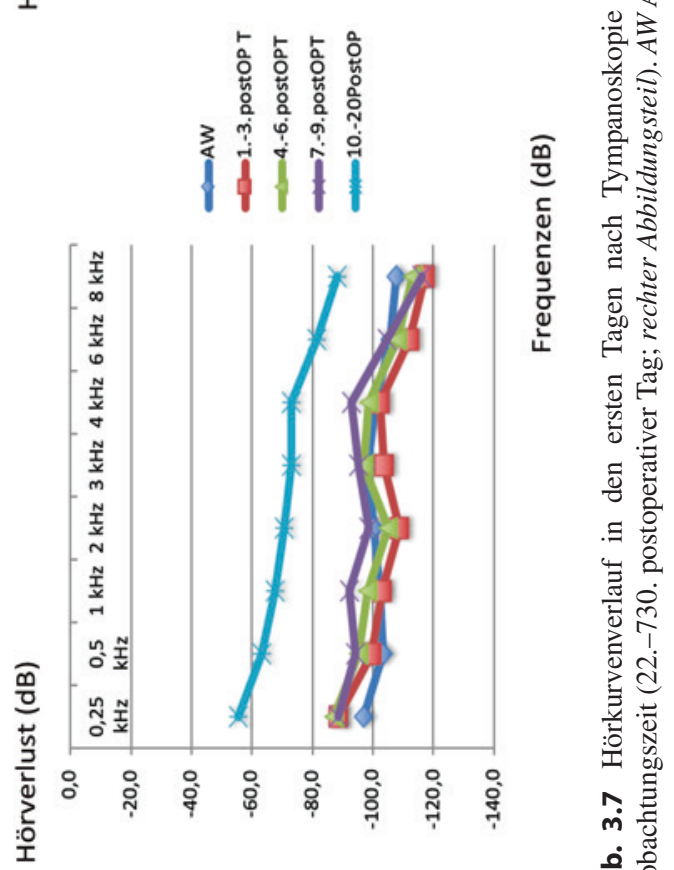

ब

N

ป

这

$=$ को

एँ

흄

$\Xi$

产

ग

อั

记贻

ค่

은 
einem Monat Abstand zur Tympanoskopie bis hin zu einem Maximalwert von 2363 Tagen (6,5 Jahre) nach chirurgischem Eingriff durchgeführt worden waren, stellten sich im weiteren Zeitverlauf nach der 3. postoperativen Woche bis im Mittel 2,3 Jahre nach chirurgischem Eingriff keine wesentlichen, allenfalls noch marginale Veränderungen im Hörvermögen ein (Abb. 3.7, rechter Abbildungsteil).

Dieses Resultat steht im Einklang mit Mertens et al., die herausgefunden hatten, dass Hörverbesserungen 19 Tage nach Tympanoskopie zu $94 \%$ und 28 Tage postoperativ zu $100 \%$ abgeschlossen waren [61]. Aufgrund ihrer Beobachtungen lag der späteste Zeitpunkt, zu welchem eine Hörverbesserung einsetzte, 11 Tage nach dem chirurgischen Eingriff. Dem widerspricht die vor vielen Jahren von Eichhorn et al. berichtete allgemeine Beobachtung, dass es auch langfristig nach Hörstürzen noch zu Hörerholungen kommen kann [15]. Dieser Bericht kann nach aktuellen Erfahrungen allenfalls auf Einzelfälle zutreffen, die insgesamt aber nicht so häufig auftreten, dass sie in statistischen Berechnungen eines größeren Patientenkollektiv den Mittelwert verändern.

Vergleicht man die Ergebnisse der Studien, die den Hörerfolg zum Zeitpunkt der Tamponadeentfernung zum im Rahmen der Nachuntersuchung erreichten Hörgewinn in Beziehung setzt, mit unserer Beobachtung, dass die größten Hörverbesserungen zwischen dem 10. und 20. postoperativen Tag eintreten, und den Erfahrungen von Mertens et al., dass am 28. postoperativen Tag das Endresultat der Hörerholung eingetreten ist, ergibt sich eine Diskrepanz, die sich in Abb. 3.8 niederschlägt. Die aus den verschiedenen Studien zu unterschiedlichen Fragestellungen entnommenen Daten weisen zusammenfassend eine Sättigungskurve auf, bei der der Haupthörgewinn dem Zeitintervall der ersten Woche nach dem chirurgischen Eingriff zukommen würde. Es bleibt also ein Klärungsbedarf

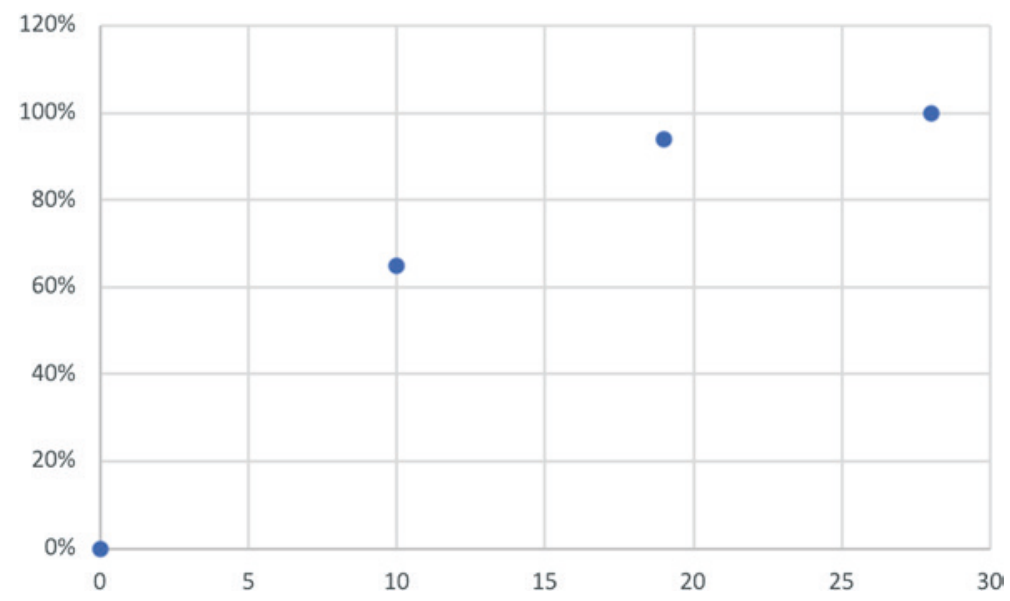

Abb. 3.8 Ausmaß der Hörerholung in Tagen nach der Tympanoskopie, dargestellt in Prozent des Gesamthörerfolges (100\%). Abszisse: Tage nach der Operation; Ordinate: Prozentsatz des Hörgewinns vom Gesamthörerfolg 
hinsichtlich der Frage, ob eher früh (1. Woche) oder verzögert (bis zur 3. Woche) nach der Tympanoskopie der Hauptanteil der Hörverbesserung erreicht wird.

\subsubsection{Hörentwicklung von der stationären Aufnahme bis zum Nachuntersuchungstermin unter zwei unterschiedlichen Blickwinkeln}

Versuch der Differenzierung des Effektes der systemischen Kortikoidbehandlung und der TympanoskopieIn Abb. 3.9 ist die Entwicklung des gemittelten Hörvermögens (PTA ${ }_{4}$ ) während des gesamten Beobachtungszeitraumes bei unseren Patienten zusammengefasst, untergliedert in eine Vielzahl von „Unterzeitintervallen“. Bei einer ersten Betrachtungsweise (linker Abbildungsteil) beginnt die Hörwerteerfassung mit der stationären Aufnahme und dem Beginn der i.v.-Kortikoidtherapie und, nach einer Unterbrechung des Kurvenzuges, folgt die Aufzeichnung mit den Zeitperioden, die der Tympanoskopiebehandlung abgestuft nachfolgen. Der Unterschied des letzten Hörwertes der präoperativen Beobachtungsperiode (erkenntlich am Kurvenunterbruch) und dem ersten Hörwert der postoperativen Beobachtungsperiode erklärt sich aus der Tatsache, dass nur von einem Teil der Patienten entweder mehr als 7 Tage nach systemischer Kortikoidgabe prästationär bzw. mehr als 20 Tage poststationär Audiogrammkontrollen vorlagen, die aber nur anteilsmäßig in die Bestimmung des Durchschnittswertes der einzelnen zeitlichen Erhebungsintervalle (prästationär, poststationär, Nachuntersuchung) eingingen. Ebenso beinhaltete der erste Hörverlust der postoperativen Untersuchungsperiode gleichermaßen nur die Hörergebnisse von einem geringen Teil der Patienten, für die insgesamt der mittlere Hörverlust in dieser Zeitperiode berechnet werden konnte. Dies führte zur zunächst schwer erklärbaren „Besonderheit“, dass die Ausgangswerte der Kurve in dem einer Unterbrechung folgenden, nächsten Zeitintervall bei einem geringeren Wert starteten, als von den aus einem reduzierten Patientenanteil gemessenen Hördaten gegen Ende des vorausgegangenen Zeitabschnittes erreicht worden war.

Im rechten Teil der Abb. 3.9 ist das Hörsturzereignis selbst als Ausgangspunkt der Betrachtung für die Entwicklung der Hörverluste von den Patienten gewählt worden, die noch am Hörsturztag in der Klinik erschienen. Die nachfolgenden Hördaten beziehen sich dann jeweils auf den zeitlichen Abstand zum Hörsturz und nicht, wie im linken Abbildungsteil, auf den stationären Aufnahmetag bzw. den OP-Termin. Bei einer derartigen Betrachtungsweise kam es zu einer langsamen, stetigen Besserung des Hörvermögen bei der die Operation - da zu individuell verschiedenen Zeitpunkten nach der Hörminderung durchgeführt - nicht mehr aus dem Kurvenverlauf heraus zu erkennen ist. Hier stellte sich dann noch einmal eine Besserung des Hörvermögens unmittelbar nach dem Therapieende zum Nachuntersuchungszeitpunkt ein. Die zuletzt gemessenen Hörverluste stimmen mit denen im linken Abbildungsteil überein.

Mit dieser letztgenannten Darstellung versuchten wir zu erreichen, dass die Therapieeffekte (i.v.-Kortikoidbehandlung und Tympanoskopie) bei der Entwicklung des Hörvermögens nach dem Hörsturz so weit wie möglich ausgeschaltet wurden. Wir sahen dabei, dass es zu einer mehr oder weniger kontinuierlich linearen, aber nur langsamen Hörverbesserung der Patienten im Laufe der Zeit gekommen war. Diese hatte jedoch auch am durchschnittlich 24. Tag nach dem Auftreten des Hörsturzes noch nicht das Ausmaß erreicht, das wir am gemittelten 15. Tag nach Tympanoskopie beobachtet hatten. Eine Restitution des Hörvermögens, wie sie im Tierversuch sogar spontan innerhalb von 3 Wochen nach der Destruktion der Rundfenstermembran nachgewiesen worden war, ließ sich bei unserem Patientengut bei einer derartigen Betrachtungsweise allenfalls in Form einer partiellen Hörerholung nachvollziehen. Wir sehen deshalb Anhaltspunkte dafür, dass man beiden Behandlungsschritten, nämlich dem der systemischen Kortikoidbehandlung und auch dem der Tympanoskopie, jeweils einen positiven Effekt zuordnen kann, wobei dieser nach der operativen Maßnahme erheblich ausgeprägter war, als er mit der alleinigen Infusionsbehandlung erreicht werden konnte. 


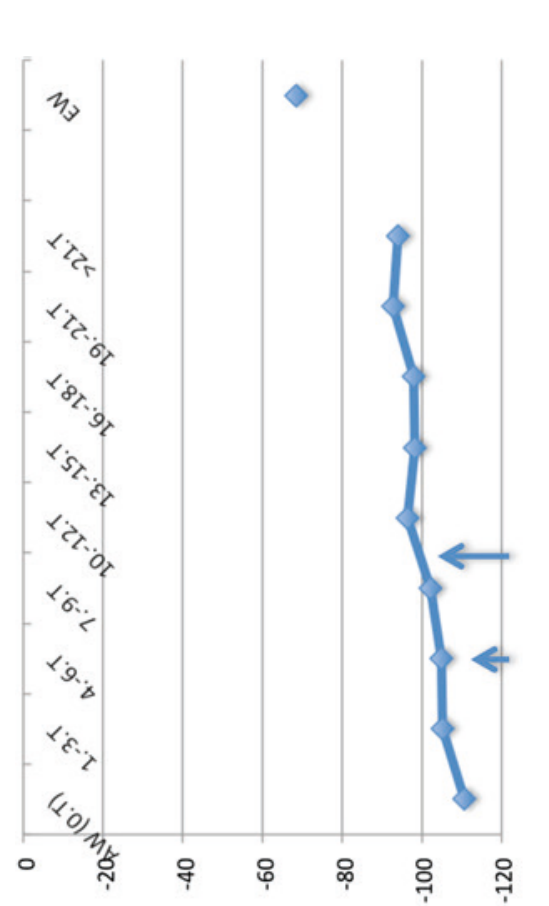

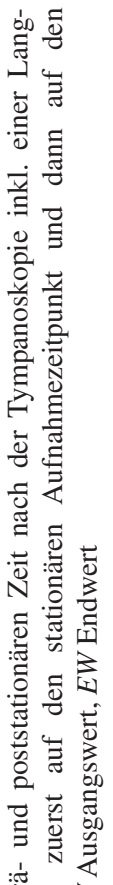

:

氖

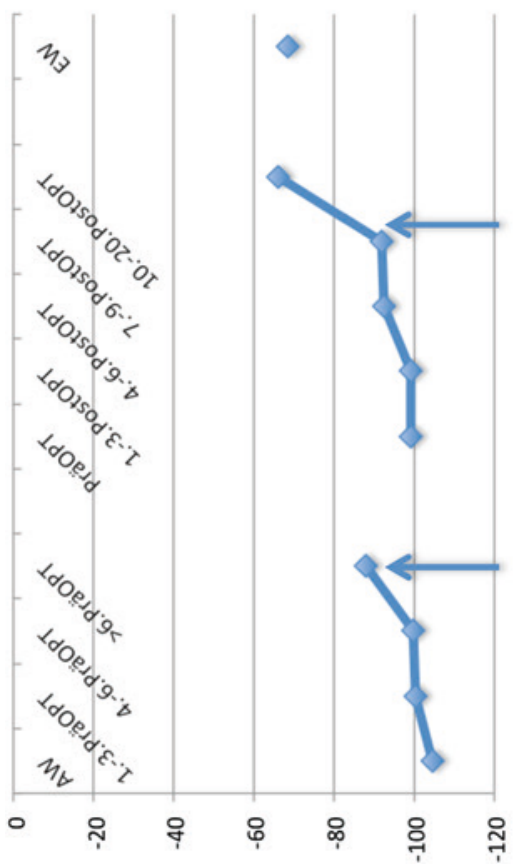

彭 范范 $-\infty$ ठ

궁:

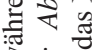

3 sे

क.

政

卷茫

a

$\theta+$

ฮี 종

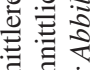

हี

के 는

on

吾 。

on

氙

o 氖 v

送总 胥 


\subsubsection{Placeboeffekt gegen Therapiewirkung der Tympanoskopie}

Es muss abschließend die Frage erlaubt sein, ob die Durchführung irgendeiner anderen, konservativen oder operativen Therapie, selbst einer Nultherapie (Placeboeffekt), zu demselben Zeitpunkt, an dem wir die Tympanoskopie durchführt hatten, nicht zu einem gleichartigen Erfolg geführt hätte, wie wir ihn mit der Mittelohroperation erzielen konnten. Schließlich lassen sich bis heute in allen vergleichbaren - wie auch in unserer Studie - die drei wahrscheinlich parallel verlaufende Heilungsprozesse (Spontanheilung; systemische Kortikoidtherapie; Tympanoskopie mit Obliteration der runden/ovalen Fensternische) nicht wissenschaftlich sauber voneinander separieren. Zum einen hat man, möglicherweise selbst in einem Kollektiv von hochgradig Hörsturzgeschädigten auch zu einem relativ späten Zeitpunkt noch von einer gewissen Quote an Spontanremissionen auszugehen, zum anderen kann sich die der Tympanoskopie mehr oder weniger lange vorausgehende und zum Teil noch nach dem Mittelohreingriff fortgeführte systemische Kortikoidtherapie anhaltend und damit etwas verzögert (nach der ersten Woche des klinischen Aufenthaltes) entfaltet haben. Insofern kann der konservative Teil der Therapie in seiner Wirkung nicht exakt von dem der Tympanoskopie abgegrenzt werden.

\subsubsection{Einschränkung der Untersuchungsaussagen}

Neben den bereits geschilderten Nachteilen muss als Einschränkung der Aussagekraft unserer Untersuchung auch angeführt werden, dass bei der Untergliederung des aus 42 Patienten bestehenden Gesamtkrankengutes Unterpopulationen mit zum Teil nur sehr geringen Fallzahlen zustande kamen (in der FL- und SLGruppe jeweils $n=10$, die dann miteinander verglichen wurden). Es ist darüber hinaus nicht sichergestellt, dass bei allen unseren Patienten mit einem hochgradigen Hörsturz in dem genannten 10-Jahres-Zeitraum auch Tympanoskopien durchgeführt wurden, wenngleich dies doch bei der überwiegenden Mehrzahl der derart Erkrankten der Fall gewesen sein dürfte.

Leider beinhaltete unsere vorliegende Studie auch kein Kontrollkollektiv, an dem die Über- resp. Unterlegenheit des durchgeführten Eingriffes gegenüber anderen, zeitgleich eingesetzten Therapieverfahren hätte evaluiert werden können. Als eine Vergleichsmethode, die zur Zeit weltweit auch als „salvage therapy“ angewandt wird, kämen hier wohl am ehesten intratympanale Kortikoidinjektionen infrage. Es wäre hilfreich, wenn in einer zukünftigen, prospektiv angelegten Folgeuntersuchung an nur einem klinischen Zentrum Patienten mit einem fortgeschrittenen Hörverlust bis hin zur Taubheit, die auf eine vorangehende systemische Glukokortikoidgabe nicht ausreichend angesprochen haben, beide Behandlungsregimes am besten verblindet nebeneinander angewendet würden. Erst in einem zweiten Schritt wäre dann der Frage der Wirksamkeit 
des frühen Einsatzes von Tympanoskopien nachzugehen, der aufgrund der bis jetzt erhobenen Daten noch nicht uneingeschränkt empfohlen werden kann. Das gleichzeitige „Mitlaufenlassen“ einer Kontrollgruppe (keinerlei medizinische Maßnahmen) oder eine weitere Fortführung einer alleinigen systemischen Kortikoidtherapie im Rahmen einer Folgestudie hätte zusätzlich den Vorteil, dass die ausgewerteten Daten in eine statistische Metaanalyse einfließen könnten, wie sie für die Behandlung mit intratympanalen Steroidinjektionen bereits in vielfältiger Weise vorliegen $[20,27,41,51,68,76,80,110,112]$.

\subsubsection{Schlussfolgerung}

Insgesamt geben unsere Auswertungen deutliche Hinweise darauf, dass die Tympanoskopie mit Obliteration des runden/ovalen Fensters eine respektable Therapiemöglichkeit bei der Behandlung der hochgradigen plötzlichen sensorineuralen Schwerhörigkeit insbesondere im Rahmen eines Second-LineEinsatzes darstellt und damit als Salvage Surgery dienen kann.

\subsection{Zusammenfassung}

Anhand eines 42 Patienten umfassenden Kollektives wurde erstens untersucht, welchen Einfluss die Latenzzeit, die zwischen einem Hörsturzereignis (Hörverlust $>60 \mathrm{~dB}$ ) und einer explorativen Tympanoskopie inkl. Abdeckung der runden und ovalen Fensternischen mit körpereigenem Bindegewebe als Behandlungsmaßnahme verstrichen war, auf die nachfolgend eintretenden Hörgewinne ausübt, und zweitens der Frage nachgegangen, welchen zeitlichen Verlauf die Erholung der Hörwerte im Tonschwellenaudiogramm ( $\left.\mathrm{PTA}_{4}\right)$ nach einer der Operation vorausgehenden systemischen Kortikoidtherapie (Second-Line-Modus) bzw. nach einer früh nach Krankenhausaufnahme (First-Line-Modus) durchgeführten Mittelohroperation nehmen kann.

Die mittleren Endhörverlustwerte unterschieden sich signifikant von den anfänglich gemessenen Hörminderungen. Auch wenn die Tympanoskopie erst mehr als 10 Tagen nach dem Hörsturz vorgenommen worden war, konnten noch deutliche Hörgewinne erreicht werden (im Mittel 37,2 dB).

Wurde der Mittelohreingriff innerhalb der ersten 6 Tage nach Hörsturzereignis durchgeführt (FL-Modus), fiel der Hörgewinn nach Operation geringer aus $(23,4 \mathrm{~dB})$ als bei Patienten, bei denen der Eingriff erst nach einem mehr als 10-tägigen Intervall vorgenommen (SL-Modus) worden war (38,1 dB). Eine präoperative systemische Steroidbehandlung hatte bis zum Operationszeitpunkt in der FL-Gruppe nach durchschnittlich 5,9 Tagen und in der SL-Gruppe 11,6 Tage nach Hörsturz noch keine deutliche Wirkung auf die anfänglich bestehende Hörminderung entfaltet.

Mit der Detamponierung des Gehörganges etwa 10 Tagen nach Tympanoskopie war aufgrund von zwei Studien das Endresultat der Hörerholung noch nicht 
erreicht. In der Folgezeit kam noch zu weiteren Hörverbesserungen, die insgesamt weitere 34,5\% des Gesamthörerfolges ausmachten.

Nach der Tympanoskopie setzte der Hörgewinn in unserem Patientengut durchschnittlich erst zwischen dem 10. und 20. Tag ein. Innerhalb der weiteren Nachbeobachtungszeit von im Mittel 2,2 Jahren zeigten sich nach der 3. postoperativen Woche keine nennenswerten weiteren Hörveränderungen mehr.

Insgesamt erholte sich der Tieftonbereich besser als der Hochtonbereich.

Die Betrachtung der Hörkurvenentwicklung anhand zweier verschiedener Zeitschienen lässt es wahrscheinlich erscheinen, dass die Hörgewinne, die nach der Tympanoskopie eintraten, nicht aufgrund einer Spontanremission, sondern eher auf die Therapieeffekte zurückzuführen sind.

Die explorative Tympanoskopie inkl. Obliteration der runden/ovalen Fensternischen zeigt respektable Ergebnisse in der Behandlung von Patienten mit einem ausgeprägten Hörsturz ( $>60 \mathrm{~dB}$ bis Surditas), insbesondere wenn der Operation eine systemische Kortikoidtherapie vorausgegangen war, sie also im SecondLine-Modus erfolgt war. Weitere Untersuchungen sollten in Studien mit Vergleichskollektiven (z. B. intratympanale Kortikoidinjektionen, Nulltherapie) den Stellenwert der verschiedenen Maßnahmen ermitteln.

Open Access Dieses Kapitel wird unter der Creative Commons Namensnennung 4.0 International Lizenz (http://creativecommons.org/licenses/by/4.0/deed.de) veröffentlicht, welche die Nutzung, Vervielfältigung, Bearbeitung, Verbreitung und Wiedergabe in jeglichem Medium und Format erlaubt, sofern Sie den/die ursprünglichen Autor(en) und die Quelle ordnungsgemäß nennen, einen Link zur Creative Commons Lizenz beifügen und angeben, ob Änderungen vorgenommen wurden.

Die in diesem Kapitel enthaltenen Bilder und sonstiges Drittmaterial unterliegen ebenfalls der genannten Creative Commons Lizenz, sofern sich aus der Abbildungslegende nichts anderes ergibt. Sofern das betreffende Material nicht unter der genannten Creative Commons Lizenz steht und die betreffende Handlung nicht nach gesetzlichen Vorschriften erlaubt ist, ist für die oben aufgeführten Weiterverwendungen des Materials die Einwilligung des jeweiligen Rechteinhabers einzuholen.

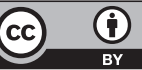

